J. Product. \& Dev., 23(3): 505- 533(2018)

\title{
QUANTITATIVE ASSESSMENT OF DESERTIFICATION IN BAHARIYA OASIS ENVIRONMENT, WESTERN DESERT, EGYPT
}

\author{
Adel A. Elwan ${ }^{1 *}$ and M.M.N. Khalil ${ }^{2}$ \\ ${ }^{1}$ Department of Pedology, Division of Water Resources and Desert \\ Lands, Desert Research Center, Cairo, Egypt \\ ${ }^{2}$ Department of Soil and Water sciences, Faculty of Agriculture, \\ Zagazig University, Zagazig, Egypt \\ *Corresponding author: Tel.: +2 01011824240, E-mail: dr.elwan_drc @ yahoo.com
}

\section{ABSTRACT}

Human activities as well as natural events can dramatically affect land degradation negatively or positively. In Egypt, agriculture is a key sector of the economy. Land reclamation seeks to transfer desert areas (New lands) to agricultural land and support the construction of new villages. The agricultural productivity improvement of these new lands is slow and requires considerable time due to their fragility and sensitivity to desertification. The main objectives of this study were the assessment, monitoring, and mapping of the areas' most sensitive to desertification in the Bahariya Oasis depression, Egypt; one of the new lands that was targeted for reclamation. Mediterranean desertification and land use (MEDALUS) approach was modified by adding new variables to the soil (SQI), vegetation (VQI), management (MQI) quality indicators while irrigation water quality (IWQI) was considered as a new indicator. The main goals were achieved via: (a) modifiyng the MEDALUS factors to obtain more reliable data at the local level, (b) identifying the most environmentally sensitive areas to desertification in the study area, c) assessing the environmental sensitivity area index (ESAI) for two time series (1984 and 2017) to determine the effects of land reclamation processes, and (d) monitoring the ESAI change between 1984 and 2017 over the studied area. Five landforms were recognized in the Bahariya Oasis depression which are alluvial plain, lowland, peneplain, plateau and hills, and playa. The ESAI results indicated that the $90.6 \%$ of the study area in 1984 was decreased to $44.2 \%$ in 2017 due to the impact of reclamation 
processes and high quality of SQI, MQI, and IWQI. By contrast, playa area as land sensitivity to desertification is increased from 15 $\mathrm{km}^{2}$ in 1984 to $25 \mathrm{~km}^{2}$ 2017, highlighting the necessity of substantial mitigation policies.

Since the Baharyia Oasis is intensively cultivated, more attention is required for the most sensitive areas to desertification in order to achieve sustainable land use in such fragile agroecosystems.

Keywords: Desertification; Sensitivity; Quality indicators; ESAI; Bahariya Oasis; Egypt.

\section{INTRODUCTION}

Land degradation (LD) is a global process which ultimately leads to a reduction or loss of the biological or economic productivity and complexity of irrigated cropland resulting from land uses or from a process or combination of processes arising from human activities and habitation patterns of soil fertility (Fantechi et al., 1995; Salvati and Bajocco, 2011). In dry areas LD, coupled with extreme biophysical and socioeconomic phenomena, may turn into an irreversible process of environmental degradation, that is desertification (Montanarella, 2007). Desertification is considered a serious problem in arid and semi-arid regions due to their fragility, and it is used to describe environmental phenomena affecting dry-lands (Salvati and Zitti, 2008). In the Mediterranean basin, sensitivity to desertification has generally been associated to ecological conditions (e.g., limited water resources, climate aridity, slope, vegetation cover, soil characteristics, and erosion) together with specific aspects of drought, human pressure (e.g., population density), and unsustainable land use management (Salvati and Zitti, 2008).

Field visits and remote sensing are suggested methods for studying land degradation. Compared to field evaluation, remote sensing data is most-effective, time-efficient, and valuable in mapping land degradation risks (Gao and Liu, 2008). Multi-spatial remote sensing data is ideal for monitoring longterm trends of land degradation and assessing land desertification severity. If combined with GIS, remote sensing can be used to identify areas of land degradation and link them to physiographic settings (Bakr et al., 2012). As environmental sensitivity is the response of the environment to a change in one or more external factors, degradation occurs when this response is deleterious the 
environment. An environmentally sensitive area (ESA) is a spatially delimited entity in which environmental and socioeconomic factors are not sustainable for that particular environment (Basso et al., 2000). The effects of environmental changes on land degradation have been studied by many researchers (Santini et al., 2010).

The Mediterranean desertification and land use (MEDALUS) approach (Kosmas et al., 1999) focuses on recognizing environmental sensitive areas (ESAs) through multi-factor approaches. For defining the environmental sensitivity area index (ESAI); soil, climate, vegetation, irrigation water, and management qualities are considered. This approach is simple, robust, widely applicable, and adaptable to new information (Kosmas et al., 1999 \& 2003). Three types of ESAs to desertification can be distinguished in the MEDALUS approach: (a) critical ESAs for areas already highly degraded, (b) fragile ESAs for areas in which any change in the delicate natural and human activity balance can lead to desertification, and (c) potential ESAs for areas threatened under significant climate change or if a particular combination of land use practices are implemented. Areas with deep to very deep, nearly flat, well drained, coarse-textured or finer soils, and under semi-arid or wetter conditions are considered non-threatened by desertification. The MEDALUS approach has been broadly used in Europe as a successful tool for detecting the most vulnerable areas to degradation (e.g. Basso et al., 2000; Contador et al., 2009; Santini et al., 2010). Although Egypt has totally different ecosystems, several studies have used the standard MEDALUS approach either on the whole Egyptian territory or in specific parts of Egyptian lands (Ali and El Baroudy, 2008; Bakr et al., 2012 and Khalifa, 2016).

Egyptian agriculture lands can be divided into old cultivated lands and new desert lands. Old lands are found in the Nile Valley as well as the Nile Delta and include the lands that have been intensively cultivated for long periods of time. New desert lands include lands that have been reclaimed relatively recently (post-1950) or are in the process of being reclaimed (UNEP, 2003). Land reclamation in the Egyptian context means converting desert areas into agricultural land by extending water canals into the desert, enhancing soil fertility, and providing infrastructure for new village construction.

In the present study, the Bahariya Oasis was chosen for assessing the ESAs to desertification. This area represents one of the newly reclaimed areas in the western desert of Egypt. Consequently, there is a 
strong need for studying the impact of the environmental sensitivity to desertification process in such a fragile agro-ecosystem.

Thererfore, the objectives of this research include: (a) identifying the most environmental sensitive areas (ESAs) to desertification in the Bahariya Oasis by modifying the MEDALUS method, (b) assessing of the most important factors affecting desertification, and (c) illustrating a GIS-based tool to monitor the ESAI change between 1984 and 2017 over the studied area.

\section{MATERIALS AND METHODS}

\section{Study site description}

Like other Mediterranean countries, Egypt shows important regional disparities in economic growth, social development, and natural resource availability. The eastern desert of Egypt have a low population density compared with the Nile valley and Delta and is considered to be potential area for establishing new communities. This is basically due to the strategic location and existence of various natural resources such as minerals, cultivable soils, and recreation places. The study was conducted in the Bahariya Oasis of the western desert, Egypt. It is located at $155 \mathrm{~km}$ west of the Nile valley and about $305 \mathrm{~km}$ to the southwest of Cairo between longitudes from $28^{\circ} 30^{\prime}$ to $29^{\circ} 10^{\prime} \mathrm{E}$ and latitudes from $27^{\circ} 45^{\prime}$ to $28^{\circ} 30^{\prime} \mathrm{N}$ (Fig. 1). It covers an area of approximately $2800 \mathrm{~km}^{2}$. Surface elevation varies from 85 to $351 \mathrm{~m}$ above sea level (Fig. 2a). The study area is a bolson (i.e., an internally drained intermontane depression; Peterson, 1981) and includes Lake playa. The Bahariya Oasis territory is an important natural depression in Egypt and is characterised by a hyper-arid climatic condition. This Oasis is a very important area from the standpoint of economics, industries, agriculture, and tourism.

Geology of Bahariya Oasis consists of the Bahariya formation, which forms the floor of the depression. Other geological formations include El-Heiz formation, El-Hefhuf formation, and Quaternary deposits (El-Sisi et al., 2002; Khalifa et al., 2006). The Bahariya Oasis, initially developed during the lower Eocene due to tectonic movements which is now occupied by a major depression floor dotted by several isolated residual hills and is surrounded by escarpments from the 


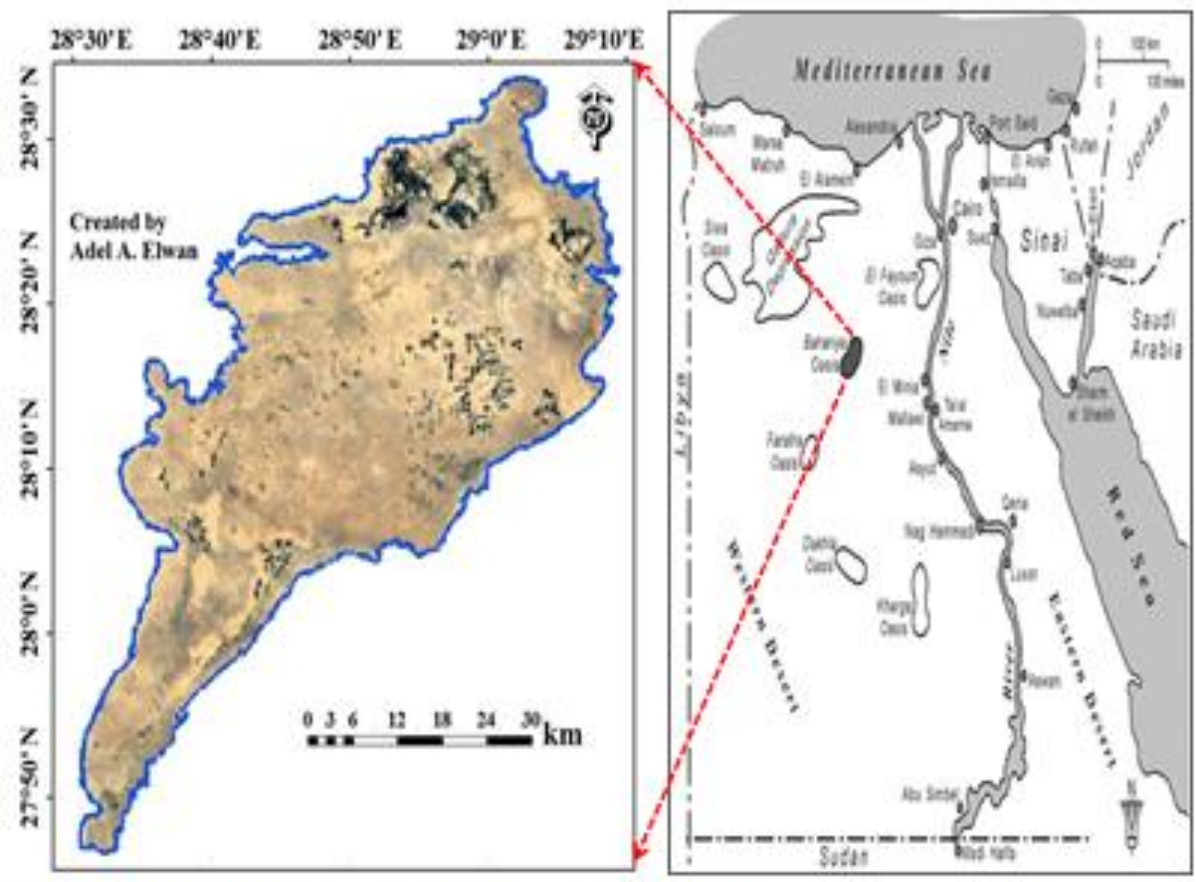

Fig. 1. Location of the Baharyia oasis depression, wesetern Desert of Egypt Left panel shows the Satellite Imajery of the oasis.

northern and eastern parts (Hamdan 2012; Masoud and El-Osta, 2016) (Fig. 2b). The bottom of the Bahariya depression is carved into the clastic Upper Cretaceous of El-Bahariya Formation. Bahariya Formation of the Lower Cenomanian, which consists of fluviatile sandstone and covers the floor of the El-Bahariya depression with a thickness of about $70.5 \mathrm{~m}$. (Friable sandy formation and calcareous clay). Surrounding escarpment is the edge which separates the depression and plateau is represented by Upper Cretaceous of Campanian age and El Hiez Formation (Fig. 2b). The plateau surface is rugged with a northward slope. It is dissected by long and short dry wadis draining into the excavated depression (Hamdan and Sawires, 2013).

Geomorphologically, the area under investigation consists of five main dominant landforms (Fig. $2 b \&$ c). These are flat to almost flat alluvial plain $\left(1585 \mathrm{~km}^{2}\right)$, almost flat lowland $\left(350 \mathrm{~km}^{2}\right)$, gently undulating to undulating peneplain $\left(590 \mathrm{~km}^{2}\right)$, moderately to highly dissected plateau with isolated conical hills distributed all over the depression of Bahariya $\left(250 \mathrm{~km}^{2}\right)$, and developed playa $\left(25 \mathrm{~km}^{2}\right)$ 


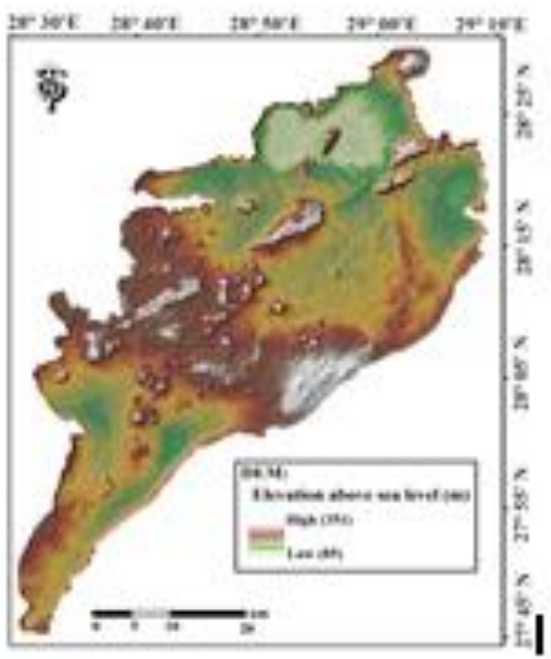

(a) Source: Modified after Blogegar (2017)

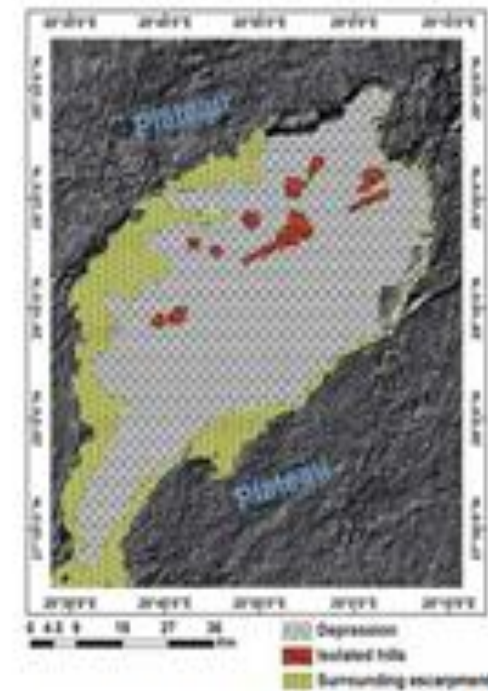

(b) Source: Mavood and El-Ora (2016)

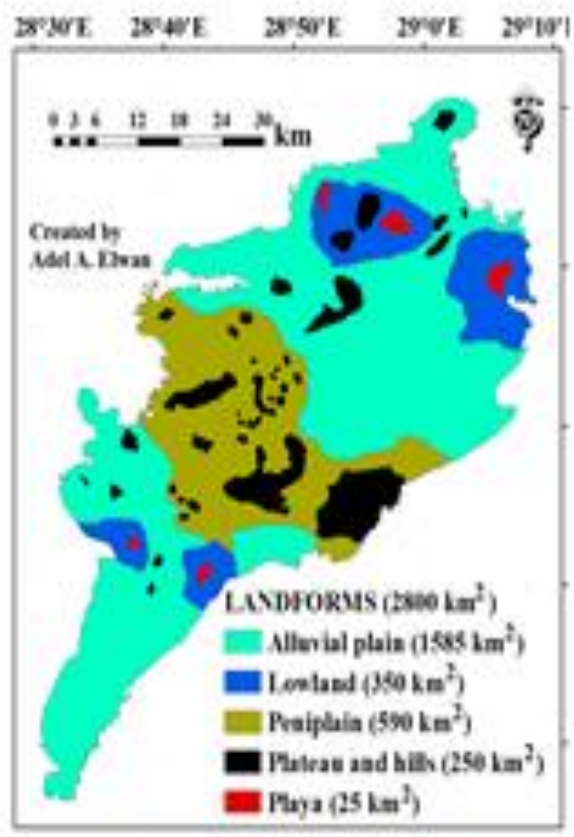

(c) Source: Own study

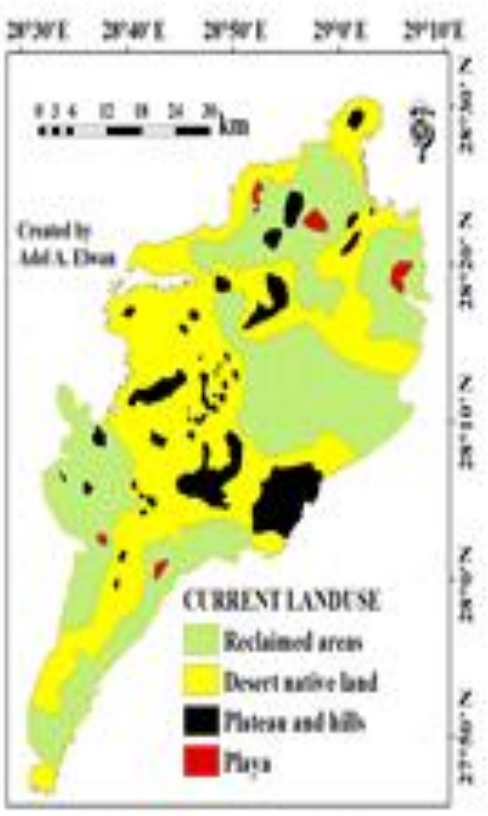

(d) Source: Own study

Fig. 2. Geomorphological information of Baharyia oasis depression (a) A general gaomorphological map. (b) A digital elevation model, (c) Detailed landforms of the oasis depression, and (d) The current landuse in the oasis. 
(Rasheed et al., 2008). In lowland, there are some villages; e.g., ElBawiti, El-Qasr, Zabu, Mandisha, and El-Ayun located in the Northern part of Bahariya and El-Heiz, Aiwon Tahblamon and El-Heiz ElBahary villages located in the southern part of Bahariya Oasis (Rasheed et al., 2010).

The main water resource for irrigating the reclaimed areas (Fig. 2d) in this Oasis is groundwater stored within the Nubian Sandstone Aquifer (NSSA) (Masoud and El-Osta, 2016). Groundwater is susceptible to anthropogenic activity resulting from urbanisation, increase in populations, land reclamation, increase of agricultural activities and lack of proper sewage and irrigation drainage systems (El-Sisi et al., 2002).

\section{Data-set}

Quality indicators were assessed via 51 soil samples collected from 15 pedons across the landforms of study area. The morphological descriptions of each horizon/layer of representative pedons and their sites were made in the field itself per the standard procedures given by FAO (2006) and Soil Science Division Staff (2017). Each sample was geo-referenced using the global positioning system. The soil samples were air-dried, ground, and passed through a 2-mm sieve. Large fragments $(>2 \mathrm{~mm}$ ) were determined, while $<2 \mathrm{~mm}$ soil was stored for further laboratory analysis. The soil $\mathrm{pH}$ and soil salinity $(\mathrm{dS} / \mathrm{m})$ were determined using a 1:1 soil/water extract, soil texture was done via hydrometer and dry sieving, and soil organic matter (SOM) content was carried out using the Walkley-Black method (Soil Survey Staff, 2014). In addition, fifteen water samples were collected from different irrigation wells (groundwater). The water samples were appropriately handled and preserved. The $\mathrm{pH}$ and water salinity $(\mathrm{dS} / \mathrm{m})$ were measured promptly and then acidified to $\mathrm{pH} 2$ for cation and anion analyses (Clesceri et al., 1998). The analysis results were used to document water quality for agricultural purposes. Soil data in 1984 were obtained from the archives of the Electronic Library, Desert Reseach Center (DRC staff, 1984).

\section{Environmental indicators}

In the MEDALUS approach (Kosmas et al., 1999), the score was allocated to each parameter in each quality index. The scores ranged 
from " $1=$ best" (for the least sensitive areas to desertification) to " $2=$ worst" (for the most sensitive areas to desertification).

The standard system as already stated by Kosmas et al., (1999), Basso et al. (2000), Sepehr et al. (2007), and Bakr et al. (2012), severe desertification rate could result from a combination of inadequate land management together with a particular set of environmental factors (soil, climate, and vegetation). A quantification of the different sensitivity levels of the territory can be carried out by evaluating over time the overall influence that single variables have on the phenomenon under study (Salvati and Zitti, 2008). A sensitivity score system was then applied, based on the estimated degree of correlation between the various factors and desertification. Four thematic indicators quantifying the environmental quality in terms of climate (Climate Quality Index, CQI), soil (Soil Quality Index, SQI), vegetation (Vegetation Quality Index, VQI) and land management (Land Management Quality Index, MQI), were estimated as the geometric mean of the different scores for each involved variable. The soil, climate, and vegetation quality indicators are related to the physical environment. However, the management quality indicator closely relates to human-induced stress on the environment (Kosmas et al., 1999). The standard MEDALUS approach is highly flexible and allows updates according to local conditions and the availability of information (Contador et al., 2009). Accordingly, new parameters were introduced by the current study to the standard MEDALUS approach including soil structure, SOM, electrical conductivity (EC), and soil $\mathrm{pH}$ for SQI and variables of applied technology, water management, soil management, crop managemnt, and labor force for MQI. Furthermore, the irrigation water quality indicator was inserted from Bakr et al. (2012) with associated parameters of $\mathrm{EC}_{\mathrm{w}}$, chloride $(\mathrm{Cl})$, and sodium adsorption ratio (SAR) according to the irrigation water quality guidelines (Ayers and Westcot, 1985). Table 1 provides a summary of the modified MEDALUS approach indicators, integrated parameters, scoring, and the data source reference for each parameter.

\section{Model building and ESAI}

The general formula that has been utilized for each aforementioned indicator is:

Index $_{i}=\left(\text { parameter }_{1} \times \text { parameter }_{2} \times \text { parameter }_{3} \times \ldots \ldots\right)^{1 / n}$ 
Table 1. Indicator's weighting system (modafied MEDALUS) adopted in this study by quality theme.

\begin{tabular}{|c|c|c|c|}
\hline Indicat & Parameters & Class and description & Weight \\
\hline \multirow{37}{*}{ SQI } & \multirow{4}{*}{$\begin{array}{l}\text { Sopegradient(9) } \\
\text { (Rosmas etal,1999) }\end{array}$} & (1) Very gentle to flat $<6$ & 1 \\
\hline & & (2) Gentle 6-18 & 12 \\
\hline & & (3) Stequ: $18-35$ & 1.5 \\
\hline & & (4) Very steep: $>35$ & 2 \\
\hline & \multirow{3}{*}{$\begin{array}{l}\text { Parent material } \\
\text { (Rosmas etcol, 1999) }\end{array}$} & 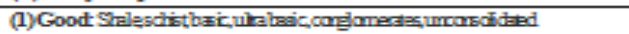 & 1 \\
\hline & & 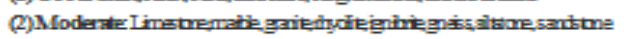 & 1.7 \\
\hline & & (3) Poor. Merl, pyroclastics & 2 \\
\hline & \multirow{3}{*}{$\begin{array}{l}\text { Drainage status } \\
\text { (Kosmasetcol, 1999) }\end{array}$} & (1) Well drained & 1 \\
\hline & & (2) Imperfectly dreined & 12 \\
\hline & & (3) Pootlydrainad & 2 \\
\hline & \multirow{4}{*}{$\begin{array}{l}\text { Soil depth } \\
\text { (Rosmasetcol, 1999) }\end{array}$} & (1) Deep: $>75 \mathrm{~cm}$ & 1 \\
\hline & & (2) Moderate $30-75 \mathrm{~cm}$ & 12 \\
\hline & & (3) Shallow: $15-30 \mathrm{~cm}$ & 15 \\
\hline & & (4) Very shallow: $415 \mathrm{~cm}$ & 2 \\
\hline & \multirow{5}{*}{$\begin{array}{l}\text { Structure } \\
\text { (Currentpaper) }\end{array}$} & (1) Gramular (soil_Aggegates) & 1 \\
\hline & & (2) Blodly, wedge & 13 \\
\hline & & (3) Prismatic, cohnmax & 15 \\
\hline & & (4) Lenticular, platy & 1.7 \\
\hline & & (5) Structureless (M Cassive single grain) & 2 \\
\hline & \multirow{4}{*}{$\begin{array}{l}\text { Texture } \\
\text { (Kosmasetoi, 1999) }\end{array}$} & (1) Good I, SCI, SI, LS, CI & 1 \\
\hline & & (2) Moderate SC,SIL, SiCI & 12 \\
\hline & & (3) Poor Si, C, SiC & 1.6 \\
\hline & & (4) Very poor.S & 2 \\
\hline & \multirow{5}{*}{$\begin{array}{l}\text { SOM }(90) \\
\text { Sepehrex } \alpha \text { (2007) }\end{array}$} & (1) Very good: $>3$ & 1 \\
\hline & & (2) Good 2-3 & 12 \\
\hline & & (3) Moderate 1-2 & 15 \\
\hline & & (4) Poor: 05-1 & 1.7 \\
\hline & & (5) Very poor: $<0.5$ & 2 \\
\hline & \multirow{5}{*}{$\begin{array}{l}\text { EC. (dS'm) } \\
\text { (Baikr etcal, 2012) }\end{array}$} & $(1)<12$ & 1 \\
\hline & & (2) $12-25$ & 12 \\
\hline & & (3) $>25-45$ & 15 \\
\hline & & (4) $>45-9$ & 1.7 \\
\hline & & (5) $>9$ & 2 \\
\hline & & (2) $5.5-6.5$ & 1 \\
\hline & & (3) $>6.5-7.5$ & 1.5 \\
\hline & & (4) $>7.5-8.4$ & 1.7 \\
\hline & & (5) $>8.4$ & 2 \\
\hline \multirow{10}{*}{ CQI } & \multirow{3}{*}{$\begin{array}{l}\text { Rainfall (mm) } \\
\text { (Kosmas et al., 1999) }\end{array}$} & (1) $>650$ & 1 \\
\hline & & (2) $280-650$ & 1.5 \\
\hline & & (3) $<280$ & 2 \\
\hline & \multirow{5}{*}{$\begin{array}{l}\text { Aridity (P/PET) } \\
\text { (Bakr et al., 2012) }\end{array}$} & (1) Humid: $>0.65$ & 1 \\
\hline & & (2) Dry sub-humid: $>0.50-0.65$ & 1.2 \\
\hline & & (3) Semi-arid: $>0.20-0.5$ & 1.5 \\
\hline & & (4) Arid: $0.05-0.20$ & 1.7 \\
\hline & & (5) Hyper-arid <0.05 & 2 \\
\hline & \multirow{2}{*}{$\begin{array}{l}\text { Slope aspect } \\
\text { (Kosmas et al., 1999) }\end{array}$} & (1) $\mathrm{NW}-\mathrm{NE}$ & 1 \\
\hline & & (2) SW-SE & 2 \\
\hline
\end{tabular}


Table 1. Contivened

\begin{tabular}{|c|c|c|c|}
\hline Suat & hrosen & 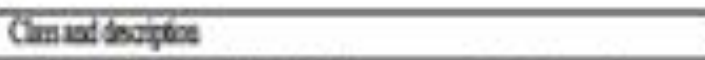 & Westis \\
\hline \multirow{18}{*}{$v$} & \multirow{3}{*}{ 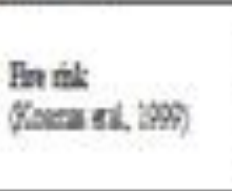 } & 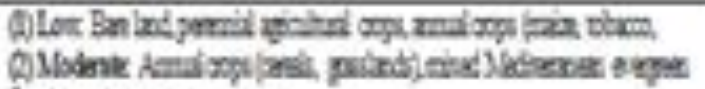 & $\begin{array}{l}1 \\
15\end{array}$ \\
\hline & & 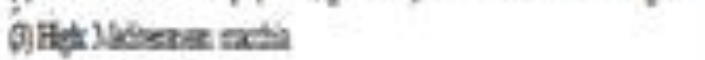 & 16 \\
\hline & & 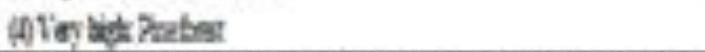 & 2 \\
\hline & \multirow{5}{*}{ 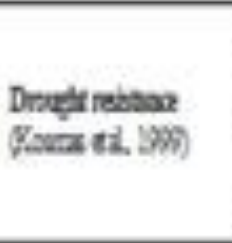 } & 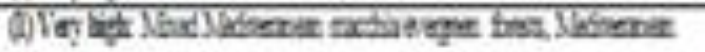 & 1 \\
\hline & & 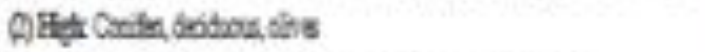 & 12 \\
\hline & & 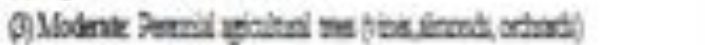 & If \\
\hline & & (A) Ler Presid paind & 17 \\
\hline & & 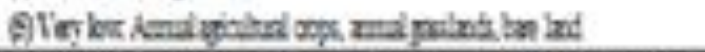 & 3 \\
\hline & \multirow{5}{*}{ 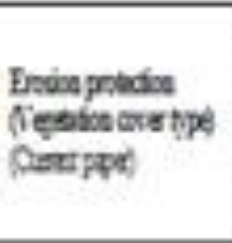 } & 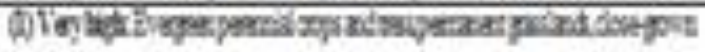 & 1 \\
\hline & & 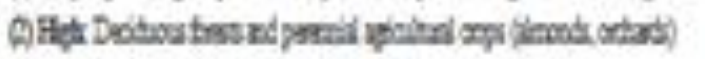 & 13 \\
\hline & & 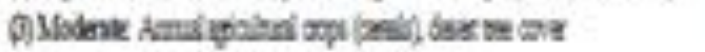 & is \\
\hline & & 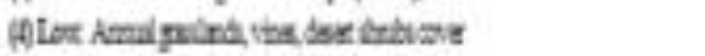 & 17 \\
\hline & & 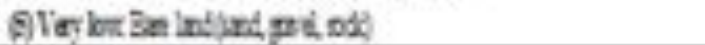 & 2 \\
\hline & \multirow{5}{*}{ 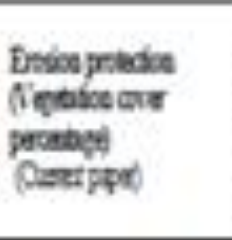 } & बiant $\times 25$ & 1 \\
\hline & & 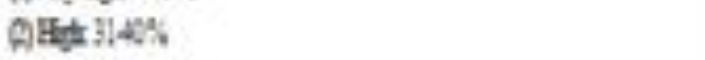 & 13 \\
\hline & & 9) Modnt $22 \%$ & 15 \\
\hline & & Aten WW & 17 \\
\hline & & Grover obs & 2 \\
\hline \multirow{11}{*}{$\operatorname{rwg}$} & \multirow{3}{*}{ 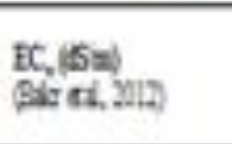 } & Q) & 1 \\
\hline & & Q) Modint 079. & 15 \\
\hline & & DEL 8 & 2 \\
\hline & \multirow{3}{*}{$\begin{array}{l}\text { Qhat } \\
\text { Bas }\end{array}$} & Q) Ler a & 1 \\
\hline & & Q) Modnte 4, & 15 \\
\hline & & ด转 $>1 \%$ & 2 \\
\hline & \multirow{5}{*}{$\begin{array}{l}\text { SAR } \\
\text { Bar calyis }\end{array}$} & Q1का & 1 \\
\hline & & Q) Ler is & 12 \\
\hline & & (9) Madanis 72 & 15 \\
\hline & & (A) $4 \mathrm{H}+120$ & 17 \\
\hline & & QVerats? & 2 \\
\hline \multirow{6}{*}{$M$} & \multirow{3}{*}{ 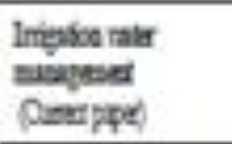 } & 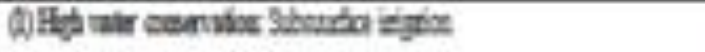 & 1 \\
\hline & & 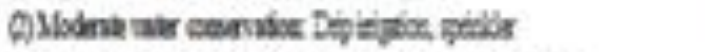 & 14 \\
\hline & & 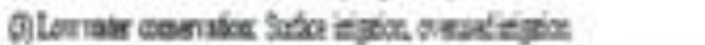 & 2 \\
\hline & \multirow{3}{*}{$\begin{array}{l}\text { Solasyesed } \\
\text { Cextppe) }\end{array}$} & 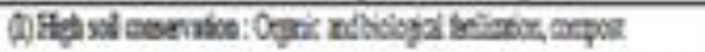 & 1 \\
\hline & & 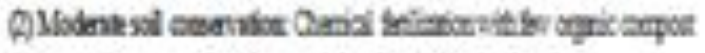 & 14 \\
\hline & & 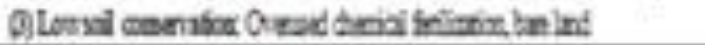 & 2 \\
\hline
\end{tabular}




\begin{tabular}{|c|c|c|}
\hline \multirow{3}{*}{$\begin{array}{l}\text { Crop management } \\
\text { (Currentpaper) }\end{array}$} & (1) Highlymechanized aulivation, ecofinindly pestmanagement & 1 \\
\hline & (2) Moderatly mechanized alliv ation, chemical pesticides & 1.4 \\
\hline & (3) Low mechanized allivation, overused agrochemicals, overgraing, bare land & 2 \\
\hline \multirow{3}{*}{$\begin{array}{l}\text { Applied technology } \\
\text { (Currentpquer) }\end{array}$} & (1)High: new tedmologies related to sol, water, and crop are applied & 1 \\
\hline & (2) Moderate: few application tedmology are applied for sol, wate, and crop. & 1.4 \\
\hline & (3) Poor no tedmologies are applied & 2 \\
\hline \multirow{3}{*}{$\begin{array}{l}\text { Social } \\
\text { (Currentpaper) }\end{array}$} & (1) High avalablity of skilledlebor $(>80 \%)$ & 1 \\
\hline & (2) Moderate avalablity of skilledlabor (50-80\%) & 1.4 \\
\hline & (3) Low avalability of skilledlabor ( $650 \%$ ) & 2 \\
\hline \multirow{3}{*}{$\begin{array}{l}\text { Institutional } \\
\text { characteristics (Policy) } \\
\text { (Kosmas etal, 1999) }\end{array}$} & (1) High degree of implementation of environmental protection policies & 1 \\
\hline & (2) Moderate degree of mplementation of envirommental protection policies & 1.4 \\
\hline & (3) Low degree of mplementation of envircrmental protection policies & 2 \\
\hline
\end{tabular}

\section{Explenations:}

SQI (Sol Quality Indicator), CQI (Climate Quality Indicator), VQI (Vegateion Quality Indicator), IWQI (Inigation Water Quality Indicator), MQI (Management Quality Indicator).

where $i$ represents the different quality indices, and $n$ represents the number of parameters.

The thematic indicators for the standard MEDALUS approach (Table 1) include; soil quality indicator (SQI) (Algorithm 1), climate quality indicator (CQI) (Algorithm 3), vegetation quality indicator (VQI) (Algorithm 3), irrigation water quality indicator (IWQI) (Algorithm 4), and management quality indicator (MQI) (Algorithm 5). The indicators were calculated as the geometric mean of the different weights of each individual parameter as:

SQI $=($ Slope $\times$ Parent material $\times$ Drainage $\times$ Soil depth $\times$ Structure $\times$

$$
\text { Texture } \left.\times \mathrm{SOM} \times \mathrm{EC}_{\mathrm{e}} \times \mathrm{pH}\right)^{1 / 9}
$$

$\mathrm{CQI}=(\text { Rainfall } \times \text { Aridity } \times \text { Aspect })^{1 / 3}$

$\mathrm{VQI}=(\text { Fire risk } \times \text { Drought Resistance } \times \text { Vegetation type } \times \text { Vegetation percentage })^{1 / 4}$

$\mathrm{IWQI}=\left(\mathrm{EC}_{\mathrm{w}} \times \mathrm{SAR} \times \mathrm{Cl}\right)^{1 / 3}$

$\mathrm{MQI}=(\text { Water manag. } \times \text { Soilmanag. } \times \text { Cropmanag. } \times \text { Technology } \times \text { Social } \times \text { Policy })^{1 / 6}(5)$

Then, the environmental sensitivity area index (ESAI) for the modified MEDALUS approach was calculated by algorithm 6:

$\mathrm{ESAI}=(\mathrm{SQI} \times \mathrm{CQI} \times \mathrm{VQI} \times \mathrm{MQI} \times \mathrm{IWQI})^{1 / 5}$

The ESAI score ranges from 1 (the lowest land sensitivity to degradation) to 2 (the highest sensitivity to desertification). According to the obtained ESAI values, four classes of the environmental sensitivity areas (ESAs) were assigned in the MEDALUS approach based on calculated ESAI; (a) critical areas (ESAI > 1.38), (b) fragile areas $(1.38>$ ESAI > 1.23), (c) potential areas (1.23 > ESAI > 1.17), and (d) non-affected 
areas $($ ESAI $<1.17)$. The score range for each quality indicator and final ESAI score are presented in Table 2.

\section{Map generation}

The digital elevation model (DEM) and major geomorphological map of the Baharyia Oasis were previously produced by and Elnaggar (2017) and Masoud and El-Osta (2016), respectively (Fig. 2a \& b). Depending on the different parameters that produced each indicator, ArcGIS 10.1 (ESRI, Redlands, CA) was used, as it offers good results with gently varying surfaces, then the area for each quality indicator was calculated. Furthermore, ArcGIS software was used to achieve the final ESAI maps and to build a model by overlaying different quality maps and using the corresponding algorithms. For the two specified time series, 1984 and 2017, the modified MEDALUS approach was applied to monitor the reclamation processes which were highly established and cropland was flourishing.

\section{RESULTS AND DISCUSSION}

In this study different indicators obtained from various sources (Kosmas et al., 1999; Sepehr et al., 2007; Bakr et al., 2012) and some variables related to SQI, VQI, and MQI added by current study were combined to quantify changes in land sensitivity to degradation. The results identified areas where sensitivity is increasing or decreasing over time, indicating as well the factors involved in this process (Basso et al., 2000). A continuous growth in the surface area classified as highly sensitive to desertification was observed over Baharyia Oasis during the study period. The values of the most important variables used for the computation of ESAI, estimated for 1984 and 2017 were reported in Table 3.

\section{Soil quality indicator (SQI)}

The variance in soils of the Oasis was related to landforms and reclamation processes. Soil texture and structure were good and poor qualities. Areas with very good textures (e.g., loamy sand, sandy loam, loam, sandy clay loam) represented the reclaimed areas (Fig. 2d), while the rest areas had poor qualities represented by peneplain soils. Studied soils were developed over different parent materials, which vary in their consolidations from soft (e.g., eolian and alluvial deposits), passing with 
Table 2. Final indicators and ESAI classes, description, and ranges for the study area.

\begin{tabular}{|c|c|c|c|}
\hline Index and the source & \multicolumn{2}{|c|}{ Quality classes and description } & Range \\
\hline \multirow{3}{*}{$\begin{array}{l}\text { SQI } \\
\text { (Kosmas et al., 1999) }\end{array}$} & \multicolumn{2}{|l|}{ (1) High quality } & $<1.13$ \\
\hline & \multicolumn{2}{|l|}{ (2) Moderate quality } & $1.13-1.45$ \\
\hline & \multicolumn{2}{|l|}{ (3) Low quality } & $>1.45$ \\
\hline \multirow{3}{*}{$\begin{array}{l}\text { CQI } \\
\text { (Kosmas et al., 1999) }\end{array}$} & \multicolumn{2}{|l|}{ (1) High quality } & $<1.15$ \\
\hline & \multicolumn{2}{|l|}{ (2) Moderate quality } & $1.15-1.81$ \\
\hline & \multicolumn{2}{|l|}{ (3) Low quality } & $>1.81$ \\
\hline \multirow{3}{*}{$\begin{array}{l}\text { VQI } \\
\text { (Current paper) }\end{array}$} & \multicolumn{2}{|l|}{ (1) High quality } & $1-1.25$ \\
\hline & \multicolumn{2}{|l|}{ (2) Moderate quality } & $1.26-1.55$ \\
\hline & \multicolumn{2}{|l|}{ (3) Low quality } & $>1.55$ \\
\hline \multirow{3}{*}{$\begin{array}{l}\text { MQI } \\
\text { (Kosmas et al., 1999) }\end{array}$} & \multicolumn{2}{|l|}{ (1) High quality } & $1-1.25$ \\
\hline & \multicolumn{2}{|l|}{ (2) Moderate quality } & $1.26-1.5$ \\
\hline & \multicolumn{2}{|l|}{ (3) Low quality } & $>1.5$ \\
\hline \multirow{3}{*}{$\begin{array}{l}\text { IWQI } \\
\text { (Bakr } \text { et al., 2012) }\end{array}$} & \multirow{3}{*}{\multicolumn{2}{|c|}{$\begin{array}{l}\text { (1) High quality } \\
\text { (2) Moderate quality } \\
\text { (3) Low quality }\end{array}$}} & $<1.15$ \\
\hline & & & $1.15-1.41$ \\
\hline & & & $>1.41$ \\
\hline \multirow{8}{*}{$\begin{array}{l}\text { ESAI } \\
\text { (Kosmas et al., 1999) }\end{array}$} & \multirow{3}{*}{ (1) Critical: } & $\mathrm{C} 3$ & $>1.53$ \\
\hline & & $\mathrm{C} 2$ & $1.42-1.53$ \\
\hline & & $\mathrm{C} 1$ & $1.38-1.41$ \\
\hline & \multirow{3}{*}{ (2) Fragile: } & F3 & $1.33-1.37$ \\
\hline & & $\mathrm{F} 2$ & $1.27-1.32$ \\
\hline & & $\mathrm{F} 1$ & $1.23-1.26$ \\
\hline & (3) Potentially affected & $\mathrm{P}$ & $1.17-1.22$ \\
\hline & (4) Not affected & $\mathrm{N}$ & $<1.17$ \\
\hline
\end{tabular}


Table 3. Percentage of surface area of quality indicators from the modafied MEDALUS approach parameters for the Baharyia Oasis depression, Egypt in 1984 and 2017.

\begin{tabular}{ll|cc|cc|cc|cc|c|c}
\hline \multirow{2}{*}{$\begin{array}{c}\text { Cla } \\
\text { Ss }\end{array}$} & \multirow{2}{*}{ Quality } & \multicolumn{2}{|c|}{ SQI } & \multicolumn{2}{c|}{ CQI } & \multicolumn{2}{c|}{ IWQI } & \multicolumn{2}{c|}{ VQI } & \multicolumn{2}{c}{ MQI } \\
\cline { 3 - 12 } & $\mathbf{k m}^{2}$ & $\mathbf{\%}$ & $\mathbf{k m}^{2}$ & $\mathbf{\%}$ & $\mathbf{k m}^{2}$ & $\mathbf{\%}$ & $\mathbf{k m}^{2}$ & $\mathbf{\%}$ & $\mathbf{k m}^{2}$ & $\mathbf{\%}$ \\
\hline $\mathbf{1 9 8 4}$ & & & & & & & & & & & \\
\hline $\mathbf{1}$ & High & - & & - & - & 2155 & 77.0 & - & - & - & - \\
$\mathbf{2}$ & Moderate & 1945 & 69.5 & 1605 & 57.3 & 370 & 13.2 & 260 & 9.3 & 260 & 9.3 \\
$\mathbf{3}$ & Low & 590 & 21.1 & 920 & 32.9 & - & - & 2275 & 81.3 & 2275 & 81.3 \\
\hline $\mathbf{2 0 1 7}$ & & & & & & & & & & & \\
$\mathbf{1}$ & High & 301 & 10.7 & - & - & 2155 & 77.0 & 350 & 12.5 & 395 & 14.1 \\
$\mathbf{2}$ & Moderate & 1634 & 58.4 & 1605 & 57.3 & 370 & 13.2 & 940 & 33.6 & 1245 & 44.5 \\
$\mathbf{3}$ & Low & 590 & 21.1 & 920 & 32.9 & - & - & 1235 & 44.1 & 885 & 31.6 \\
\hline
\end{tabular}

friable (e.g., mudstone, friable sandstone, and limestone) to moderately coherent (e.g., hard sandstone and limestone). Alluvial plain landform is sandy and nearly level sediments. The elevation ranges from 85 to 351 $\mathrm{m}$ based upon the digital elevation model (DEM) (Fig. 2b). According to slope analysis, the slope gradient varies from flat $(<6 \%)$ in alluvial plain to gentle (6-18\%) in peneplain landform which is associated with isolated hills. Soils varied from shallow $(15-30 \mathrm{~cm})$ in peneplain landform to deep soils $(>75 \mathrm{~cm})$ in alluvial plain. Deep soils were mostly associated with the transported parent materials (aelion and alluvial deposits) and the shallow were associated with the presence of hard pans (i.e., petrocalcic and petrogypsic horizons or duripans) or high water table. Drainage in the Oasis varied between moderate and good. Good drained areas were found in alluvial plain while moderately drained soils were observed in lowland and peneplain. By contrast, the poorly drained areas like water logged soils and playa areas were excluded. Based on the field observations, there was no drainage systems in the reclaimed areas and these areas were drained through surface channels into the developed playa. This could result from an increase in playa land from $15 \mathrm{~km}^{2}$ in 1984 to $25 \mathrm{~km}^{2}$ in 2017 , and caused the development of water logged areas and soil degradation due to salt accumulation with increasing the agricultural expansion projects in the lowland. As demonstrated by our results, land sensitivity in the lowland by increasing playa area tends to worsen over time (Figures 3a $\& b)$, highlighting the necessity of substantial mitigation policies. 
Nine variables were used to evaluate the soil quality in the Bahariya Oasis according to their impacts on desertification level. These variables are slope gradient, parent material, drainage status, soil depth, soil structure, soil texture, SOM, soil salinity, and $\mathrm{pH}$ as represented in Table 1. Variables including soil texture, depth, slope, and parent material, regarded as proxies for additional soil structure influencing factors (e.g. organic matter, compaction) are determined by the joint action of "pedogenesis factors" that are macroclimate, organisms, morphology, and time. In current case study, considering the examined time span, these variables can be considered as static, as they change slowly or rarely and by their nature are infrequently measured or mapped (Basso et al., 2000). Other variables like soil structure, SOM, soil salinity, and $\mathrm{pH}$ are highly dynamic which related to land cover changes and managment. Table 3 shows the quality indicators' area coverage of the study area according to the modified MEDALUS approach for 1984 and 2017. In 1984, the SQI results indicate that $69.5 \%$ and $21.1 \%$ of the study area had a moderate and low soil quality, respectively (Fig. 3a), while the rest of the study area was excluded as rocky or playa areas. On the other hand, the SQI values were modified in 2017 to $10.7 \%, 58.4 \%, 21.1 \%$ for high, moderate, and low, respectively (Fig. 3b) which may be attributed to enhanced SOM, soil structure, and soil salinity. Although the soil quality related parameters are inherent soil characteristics (Bakr et al., 2012), the SQI values were different in 1984 from 2017 that are highly affected with the reclamation processes. The SQI results show that $10.7 \%$ of the Oasis area $\left(301 \mathrm{~km}^{2}\right)$ has a high soil quality in 2017 compared with 0\% in 1984 (Table 3; Fig. 3). This increase in the high soil quality class occurred after adding the structure, SOM, EC, and $\mathrm{pH}$ parameters to the SQI that can greatly affect physiochemical and biological indicators of soil quality due to the suitable cultivation practices. Fig. $3 b$ shows that high soil quality areas occupy mainly the northeastern parts of alluvial plain.

\section{Climate quality indicator (CQI)}

Climate quality was described in the MEDALUS framework by the annual rainfall rate, aridity index (defined as the ratio between rainfall and reference evapotranspiration, both measured over a long-term), and aspect (Basso et al., 2000). These indicators were calculated using basic information available in the Egyptian Meteorological Authority (EMA, 2012). The findings in Table 3 showed that the CQI was the same in 

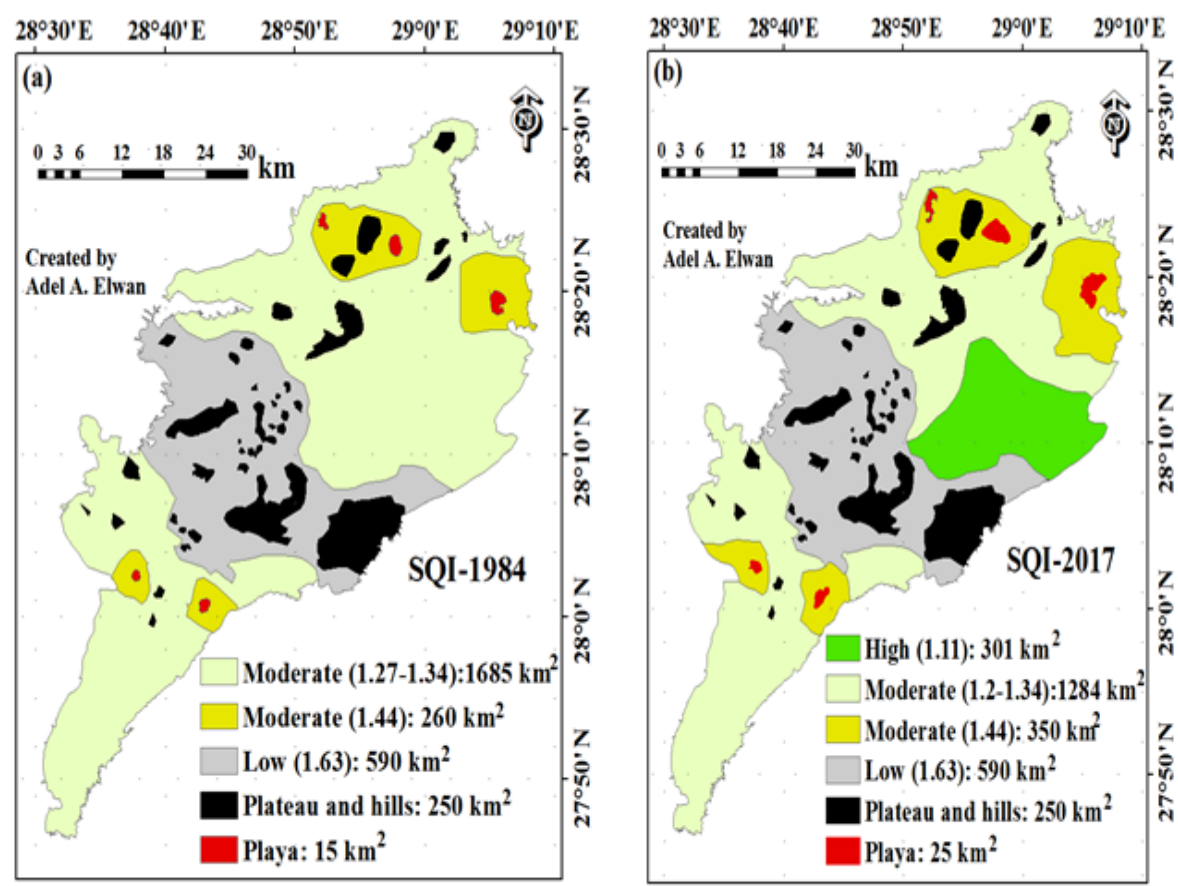

Fig. 3. Soil quality indicators for the Baharyia oasis depression. (a) SQI in 1984, (b) SQI in 2017.

1984 and 2017. Across the 1984-2017 period, the Bahariya Oasis is considered as an hyper-arid region, where the total annual long-term precipitation is about $4 \mathrm{~mm}$. The mean annual long-term temperature is 14.25. Maximum air temperature varies from $20.1^{\circ} \mathrm{C}$ in winter to $36.9^{\circ} \mathrm{C}$ in summer, with mean value of $28.5^{\circ} \mathrm{C}$. Minimum air temperature ranges between $4.9^{\circ} \mathrm{C}$ in winter and $21.0^{\circ} \mathrm{C}$ in summer, with mean value of $12.95^{\circ} \mathrm{C}$ (EMA, 2012). Accordingly, there was no observed difference between the climatic parameters of 1984 and 2017. Thus, the CQI distribution across the study area was the same in the two time series (Fig. 4a). The CQI values of Bahariya Oasis territory during the study period varies from 1.59 (moderate quality) in the northern portion to 2.0 (low quality) in the southern portion. Since the annual precipitation and aridity index are constant across the area, the only (slight) change in the CQI values was due to slope aspect (Kosmas et al., 1999). Climate quality has a great influence on the vulnerability of land to desertification due to its critical impact on the growing of vegetation and soil erosion. 

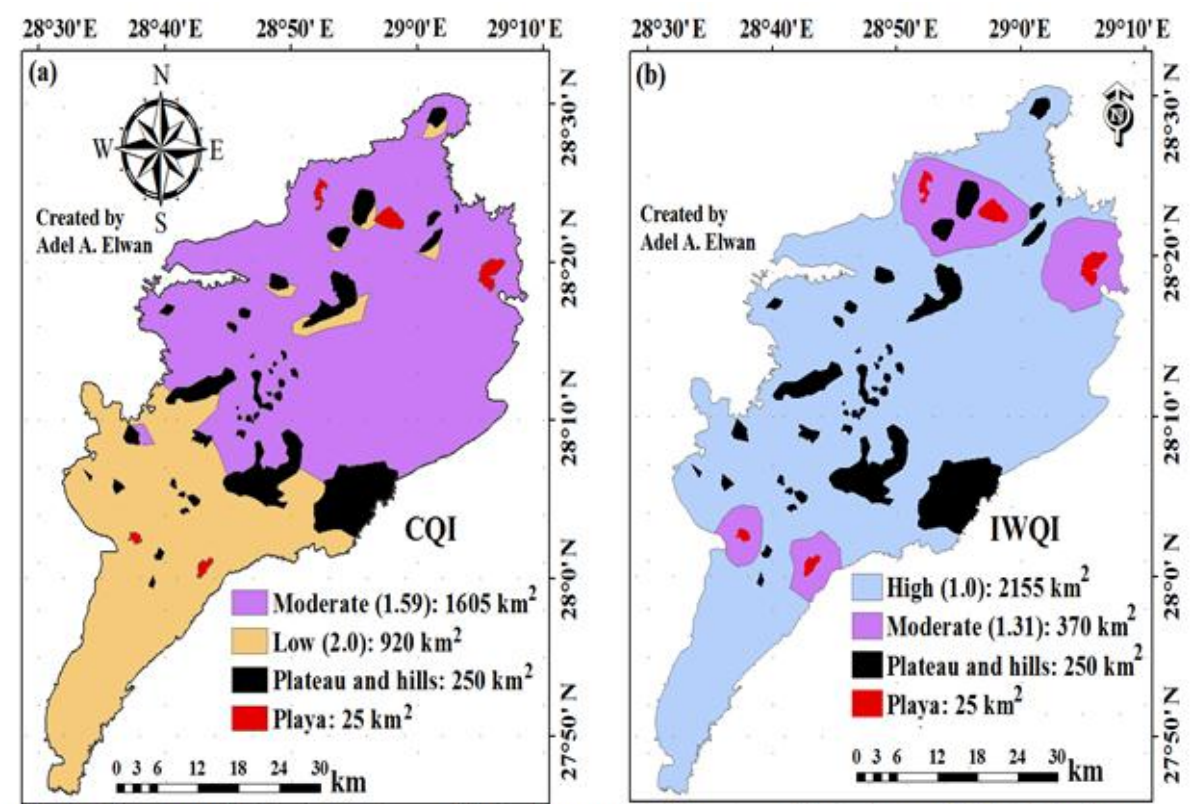

Fig. 4. Climate and irrigation water quality indicators. (a) Climate quality indicator (CQI) in 1984 and 2017, (b) Irrigation water quality indicator (IWQI) in 2017

\section{Irrigation water quality indicator (IWQI)}

Nowadays, groundwater gave a great interest, especially for use in agricultural activities. Groundwater represents the main water resource in Bahariya Oasis. The groundwater bearing strata in Bahariya Oasis belongs to the Nubian aquifer, which represents one of the largest groundwater systems within the eastern Sahara in northeast Africa. It extends under Egypt, the eastern parts of Libya, the north and northeastern parts of Chad as well as the north and northwestern parts of Sudan. The Nubian aquifer is generally a closed system. However, only in the southeastern parts there is a groundwater inflow from the Blue Nile/Main Nile rift system (Elnaggar, 2014). The main water-bearing succession in the Bahariya Oasis belongs to the Early Cenomanian and Pre-Cenomanian times (Korany, 1984; Shehata, 1992). The thickness of water-bearing sandstone in Bahariya Oasis ranges between 400 and $1400 \mathrm{~m}$. The flow direction of groundwater in the Oases is generally from the southwest to the northeast (El-Shazly et al., 1991). The chemical composition of groundwater is mainly affected by the mineralogical composition of the water bearing strata. Also, the movement of groundwater within the aquifer increases the dissolution of major ions normally become more dominant (Korany, 1984). In 
Bahariya Oasis there are some springs in addition to shallow and deep drilled wells. Most of agricultural areas and villages in the Oasis are spotted around these points. In the last decade, Bahariya depression was suggested as a proposed area for agriculture reclamation projects due to its location and the availability and good quality of groundwater (Elnaggar, 2014; El-Sisi et al., 2002).

The IWQI was included in the modified MEDALUS approach in order to assess the quality of water that was used for irrigation in the Bahariya Oasis depression. Table 3 and Fig. 4b show that $77 \%$ of the Oasis depression $\left(2155 \mathrm{~km}^{2}\right)$ has the highest IWQI value (1) indicating high irrigation water quality and only $13.2 \%$ of the study area $\left(370 \mathrm{~km}^{2}\right)$ was irrigated with moderate quality water that has a 1.31 IWQI score. Fig. $4 \mathrm{fb}$ shows that the moderate quality class was distributed in the middle of lowland due to the presence of playa which was used as a natural agricultural drainage and developed as a result of the high load of agrochemicals from fertilizers and pesticides.

\section{Vegetation quality indicator (VQI)}

The impact of land cover changes on desertification rate was assessed through the natural vegetation and cultivated crops. Vegetation cover plays an important role in mitigating the effects of desertification and land degradation phenomena. The percentage of vegetation is a function of both man-made agriculture and natural vegetation coverage. The percentage of vegetation cover is a necessary input in a multicriteria model to assess the vegetation quality index. In this research, four standard variables are considered to calculate the VQI, that are: fire risk and drought resistance of vegetation suggested by (Kosmas et al., 1999) and protection from soil erosion by vegetation cover type and percentage which suggested by the current study. Native vegetation in the lowland of Bahariya depression, which commonly associated with dry salt marshes, could be divided into four communities dominated by Sporobolus spicatus, Alhagi maurorum, Desmostachya bipinnata and Tamarix nilotica. The last three communities represent more than $80 \%$ of the natural vegetation of the Oasis (Zahran and Willis, 2009). On the other hand, the cultivated crops were dominated by date palm (Phoenix dactylifere) in the lowland and vegetables and fruits in the alluvial plain.

In 1984, the Baharyia depression was mostly bare soil with few to common natural vegetation. Accordingly, it was expected that $81.3 \%$ of the area $\left(2275 \mathrm{~km}^{2}\right)$ had low vegetation quality (VQI score: $1.61-1.80$ ) 
in peneplain and alluvial plain and only $9.3 \%\left(260 \mathrm{~km}^{2}\right)$ had moderate quality in lowland (Table 3; Fig. 5a), as the final VQI results are highly affected by the vegetation cover parameter. In 2017, the VQI results were changed due to the reclamation processes and agricultural activities. Consequently, different VQI classes were easily recognized in 2017 indicating that $12.5 \%\left(350 \mathrm{~km}^{2}\right), 33.6 \%\left(940 \mathrm{~km}^{2}\right)$, and $44.1 \%$ $\left(1235 \mathrm{~km}^{2}\right)$ of the area were assigned as high (1.24), moderate (1.31.44), and low quality (1.68-1.8), respectively (Table 3; Fig5b). Fig. 5 shows that the lowest VQI score (1.80) during the investigated period (1984-2017) occurred in the whole peneplain landform located at the middle Oasis (Fig. 5a \& b), while the highest VQI score (1.24) was observed in the northeastern part of alluvial plain (Fig. 5b) which converted to crop cover.

\section{Land management quality indicator (MQI)}

Six variables which related to the scientific management, social, and institutional characteristics were used to asses the MQI. Besides the policy variable wich suggested by Kosmas et al., (1999), five parametrs are recently added by the current study which are: irrigation water management, soil management, crop management, applied technology, and availability of skilled labors. Similar to the VQI case, the results of MQI in 1984 indicate that $81.3 \%$ of the area was categorized as the lowest management quality (Table 3; Fig. 6a) since most of the Baharyia depression was a virgin desert land with no applied management strategies. By 2017, intensive agriculture was existed with low fertility of natural resources and actions such as highly mechanized cultivation, appropriate use of biological fertilizers and ecofriendly pesticides as well as conservation measures of water, soil, and crop that enhance the productivity were considered. The results of the MQI in 2017 indicate that $31.6 \%$ of the Baharyia depression area $\left(885 \mathrm{~km}^{2}\right)$ still suffered from poor or low management quality related to fragile ecosystems, $44.5 \%$ (1245 km2) has moderat quality score (1.40), and only $14.1 \%\left(395 \mathrm{~km}^{2}\right)$ has high MQI score (1.18) (Table 3). Fig. 6b explains the majority of moderate management quality across the whole area. The results of VQI and MQI reflect their critical role in influencing the ESAI. Those aforementioned results demonstrate how the proposed methodology, 


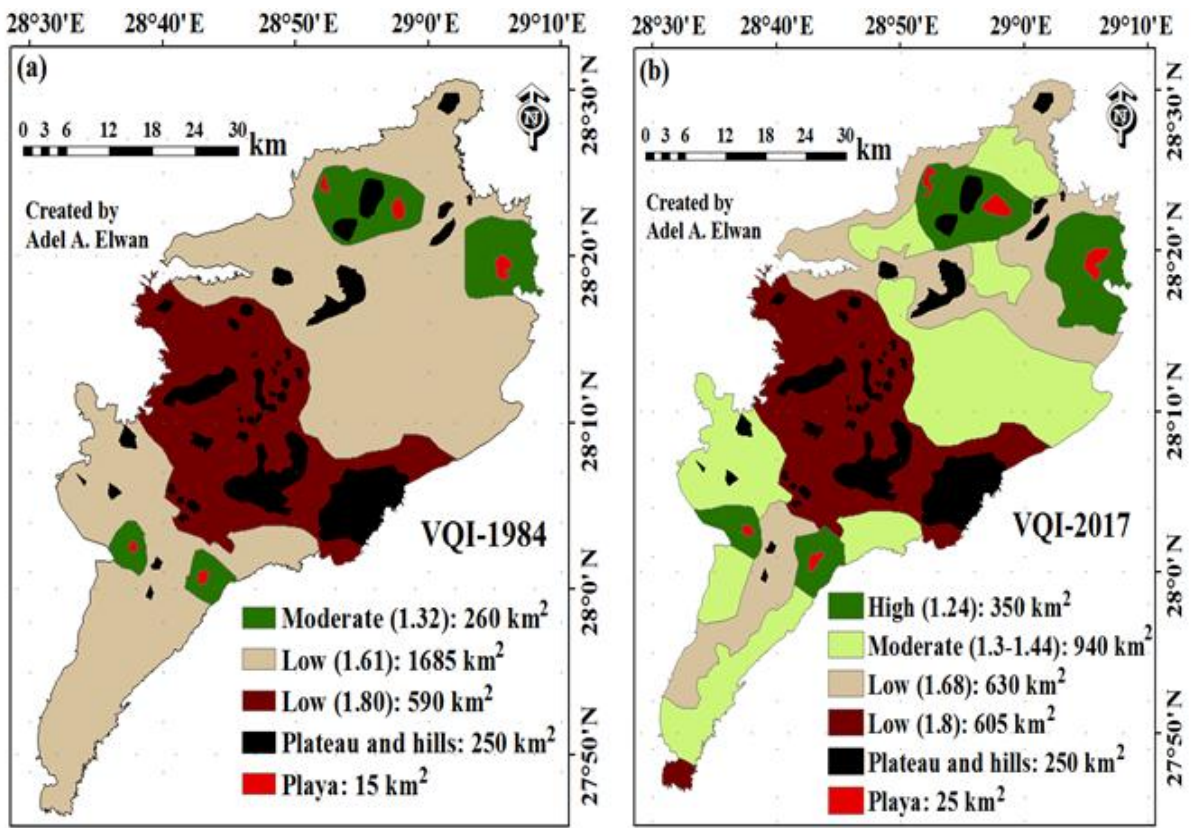

Fig. 5. Vegetation quality indicators for the Baharyia oasis depression over the period 1984-2017. (a) VQI in 1984, (b) VQI in 2017.

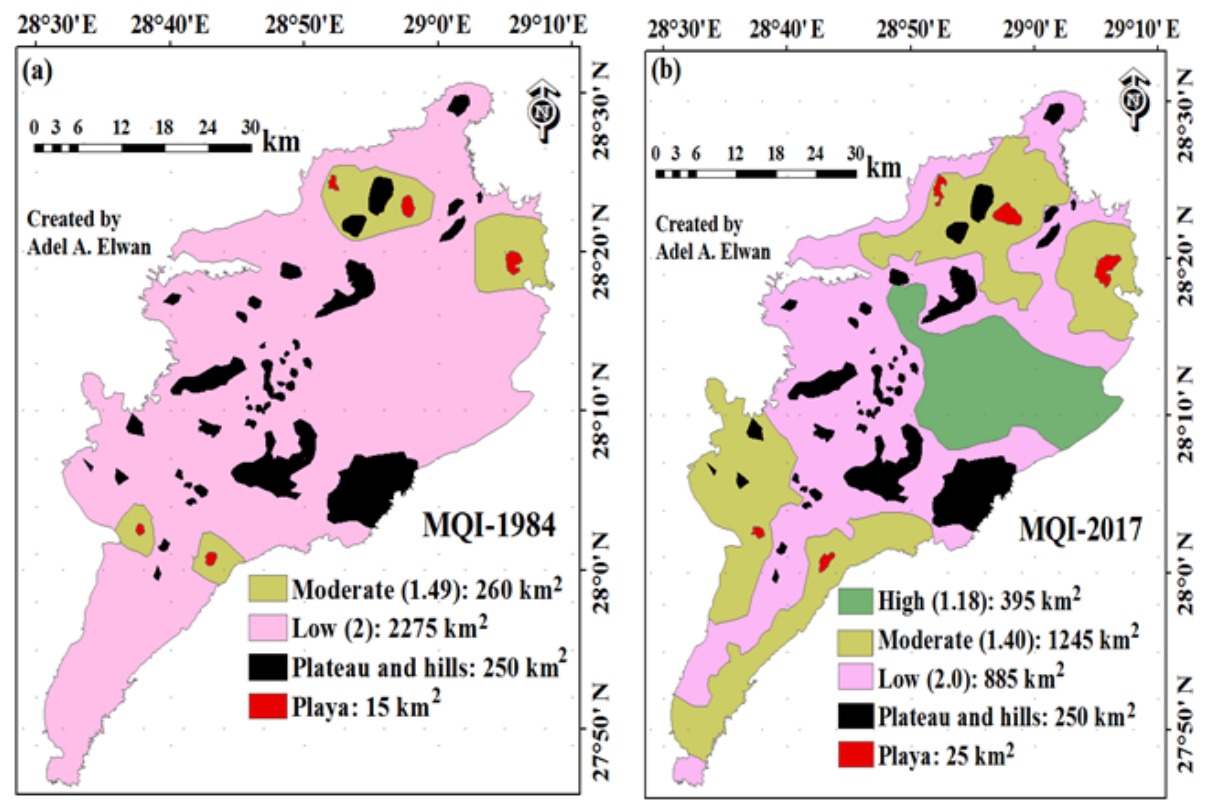

Fig. 6. Quality indicators of land management across the long-term period. (a) MQI in 1984, (b) MQI in 2017. 
using the madified MEDALUS approach, can greatly affect the final results of the quality indicators, for the SQI, IWQI, and MQI, and subsequently the estimation of ESAI to provide more reliable results depending upon the local conditions.

\section{Environmental sensitivity area index (ESAI)}

The ESAIs were calculated based on the overlaying technique of the different quality indicators which allows for the identification of links between those indicators and their spatial patterns. In agreement with Contador et al. (2009) and Bakr et al., (2012), the distribution of ESAI over the study area is closely related with the relationship between the parameters that were used to build the index. Table 4 shows the areal coverage of the ESAI for the Baharyia Oasis depression, by square kilometer and percentage using the modified MEDALUS approach over the investigated period (1984-2017). In 1984, the results reveal that almost $90.6 \%$ of the study area fell into the critical sub-classes: C1 (260 $\left.\mathrm{km}^{2}\right), \mathrm{C} 2\left(1685 \mathrm{~km}^{2}\right), \mathrm{C} 3\left(590 \mathrm{~km}^{2}\right)$ (Table 4). These results were anticipated since the study area was barren land without any strategy plan or plant coverage at this time. Fig. 7a shows that the whole area was classed as critical (C) with no appearance of the other classes. By contrast, the critical class area coverage in 2017 decreased to occupy $44.2 \%$ and the good sensitivity classes appeared. For 2017, the results show that $14.1 \%\left(395 \mathrm{~km}^{2}\right), 19.4 \%\left(545 \mathrm{~km}^{2}\right), 12.5 \%\left(350 \mathrm{~km}^{2}\right), 44.2 \%$ $\left(1235 \mathrm{~km}^{2}\right)$ of the study area were classed as not affected $(\mathrm{N})$, potentially ffected $(\mathrm{P})$, fragile $(\mathrm{F} 2)$, and critical (C1 and $\mathrm{C} 3)$ ESAs, respectively (Table 4). Fig. 7b displays the spatial distribution of ESAI over the study area in 2017. The distribution of ESAIs from Fig. 7a in 1984 or $7 \mathrm{~b}$ in 2017 perfectly matches with the quality indicator results (Figs. 3 to 6) since the highest sensitivity areas to desertification are the lowest quality areas. In general, the ESAI maps of the Baharyia depression indicate that SQI, VQI, and MQI were the most important indicators affecting the final results over the investigated long-term period from 1984 to 2017. This conclusion is consistent with the results of Sepehr et al. (2007). Furthermore, when comparing the modified MEDALUS approach in 1984 and 2017, the findings indicate that the IWQI and the parameters that were added to the SQI play an important role in decreasing the sensitivity to desertification. Thus, using modern irrigation systems, improving water management practices and 
Table 4. Distribution of ESAI by year and estimated surface of sensitive areas to desertification in the Baharyia Oasis depression, Egypt.

\begin{tabular}{ll|cc|cc|}
\hline \multirow{2}{*}{ Class } & \multirow{2}{*}{ Sub-class } & \multicolumn{2}{|c|}{ ESAI 1984 } & \multicolumn{2}{c|}{ ESAI 2017 } \\
\cline { 3 - 6 } & & $\mathbf{k m}^{\mathbf{2}}$ & $\mathbf{\%}$ & $\mathbf{k m}^{\mathbf{2}}$ & $\mathbf{\%}$ \\
\hline Not affected & $\mathrm{N}(<1.17)$ & - & - & 395 & 14.1 \\
Potentially affected & $\mathrm{P}(1.17-1.22)$ & - & - & 545 & 19.4 \\
Fragile & $\mathrm{F} 2(1.27-1.32)$ & - & - & 350 & 12.5 \\
& $\mathrm{C} 1(1.38-1.41)$ & 260 & 9.3 & 570 & 20.4 \\
Critical & $\mathrm{C} 2(1.42-1.53)$ & 1685 & 60.2 & - & - \\
& $\mathrm{C} 3(>1.53)$ & 590 & 21.1 & 665 & 23.8 \\
\hline Excluded areas & - & $\mathbf{2 6 5}$ & $\mathbf{9 . 4}$ & $\mathbf{2 7 5}$ & $\mathbf{9 . 8}$ \\
\hline Total & - & $\mathbf{2 8 0 0}$ & $\mathbf{1 0 0}$ & $\mathbf{2 8 0 0}$ & $\mathbf{1 0 0}$ \\
\hline
\end{tabular}

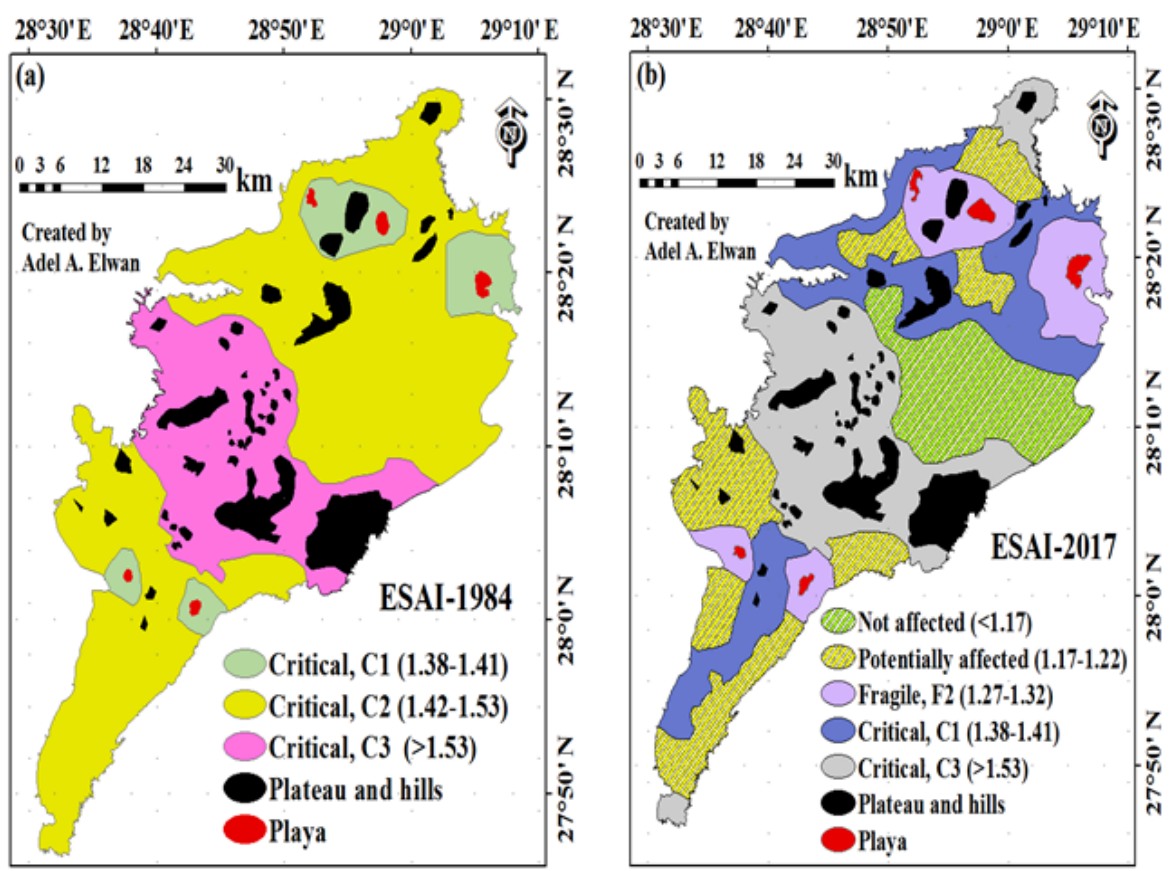

Fig. 7. Classification of the study area in terms of sensitivity to desertification.

(a) distribution of ESAI in 1984 classified as 'critical sub-classes',

(b) The current environmental sensetivity areas to desertification in 2017.

enhancing marginal land management will greatly combat the desertification process as reported by Abahussain et al. (2002).

In light of these results, a special attention should be paid in the reporting of the ESAI at adequate temporal and spatial scales. The 
possible choices should be evaluated taking into account that outputs of the model should be given at scales suitable for policy makers and other stakeholders acting at different levels (national, regional, and local). Although the ESAI was developed over several Mediterranean areas making use of extensive datasets under the framework of the MEDALUS research project, there is certainly scope for improvements. This is especially relevant as far as specific degradation processes, which are active at the local scale, are concerned. This may be possible by modifying slightly the set of variables contributing to ESAI, and by collecting long time series of suitable indicators; in fact, as this study demonstrates, a temporal approach of the phenomenon allows to better indicate the spatial direction of potential desertification processes (Salvati and Zitti, 2008). Furthermore, providing spatial information on the rate of desertification and on the variation of its anthropogenic causes may represent a kin tool to drive political actions capable to effectively contrast processes of desertification at regional scale (e.g. Briassoulis, 2004). Based on these considerations, the modified MEDALUS approach is considered as a scheme for a multi-scale decision support system which aimed at monitoring, over Mediterranean conditions, both the sensitivity to desertification and the actual processes of land degradation in a dynamic environmental and socio-economic context. The approach is modular and allows great flexibility at lower scales, while identifies a common standard working at higher scales.

\section{CONCLUSIONS}

Identifying the appropriate parameters as well as choosing the suitable spatial and temporal scale, are essential for correctly identifying the ecosystem and assessing its sensitivity to desertification. In this case study, the modified MEDALUS approach was used in two time periods (1984 and 2017) to evaluate the impacts of the reclamation processes that take place in the Baharyia Oasis depression, Egypt. The modified MEDALUS approach was applied by adding new parameters to the SQI, VQI, and MQI as well as extending the quality indicators to include an IWQI. The results clearly elucidate the role that humans play in accelerating, slowing, or eliminating desertification processes. In a fragile, vulnerable agro-ecosystem such as the Baharyia Oasis, high sensitivity to desertification exists. Thus, decision-makers should give more attention to the most sensitive areas to desertification. Results of this study show that plant cover, management, and irrigation water 
quality dramatically impact desertification processes. Access to suitable irrigation water may remain problematic issue. However, the management of such areas can be improved much more easily. Moreover, the monitoring of desertification processes over long periods of time provides valuable information and is highly recommended for proper land use planning as well as sustainable development.

This paper highlights the potential of the modified MEDALUS approach as a ESA framework to be used in the process of selecting areas needing measures for reducing or even reversing their degradation trend over time. This framework does not address specific desertification processes, but instead indicates what areas need further monitoring and attention from policy makers and stakeholders. The study also provides an example of environmental reporting based on the differences in the ESAI measured over a defined time period and calculated at the regional scale. The ESAI values estimated by year in each spatial domain, and the changing surface area of the most sensitive class (i.e. the 'critical' class), are used as a synthesis of the obtained results. Of course, other synthetic values are potentially meaningful and could be used in ESAI reporting. We believe that the flexibility and readability of the ESAI procedure makes the methodology suitable for different scale approaches and even for (small) changes in the set of input variables. Furthermore, the adaptability of the ESAI to different socio-environmental contexts makes the index applicable at the level of the whole Mediterranean basin.

\section{REFERENCES}

Abahussain, A.A., Abdu, A., Al-Zubari, Sh., Alaa El-Deen, W.K., Abdul-Raheem, N.M., 2002. Desertification in the Arab Region: analysis of current status and trends. J. Arid Environ., 51, 521-545.

Ali, R.R., El Baroudy, A.A., 2008. Use of GIS in mapping the environmental sensitivity to desertification in Wadi El Natrun depression, Egypt. Aust. J. Basic Appl. Sci., 2, 157-164.

Ayers, R.S., Westcot, D.W., 1985. Water Quality For Agriculture. FAO, Irrigation and Drainage, Paper 29, Rome.

Bakr, N., Weindorf, D.C., Bahnassy, M.H., El-Badawi, M.M., 2012. Multi-temporal assessment of land sensitivity to desertification in a fragile agro-ecosystem: Environmental indicators. Ecol. Indicat., 15, 271-280. 
Basso, F., Bove, E., Dumontet, S., Ferrara, A., Pisante, M., Quaranta, G., 2000. Evaluating environmental sensitivity at the basin scale through the use of geographic information systems and remotely sensed data: an example covering the Agribasin-Southern Italy. Catena, 40, 19-35.

Briassoulis, H., 2004. The institutional complexity of environmental policy and planning problems: the example of Mediterranean desertification. Journal of Environmental Planning and Management, 47, 115-135.

Clesceri, L.S., Greenberg, A.E., Eaton, A.D., 1998. Standard Methods for the Examination of Water and Wastewater, $20^{\text {th }} \mathrm{ed}$. American Public Health Association, American Water Works Association, Water Environment Federation, United Book Press Inc., Baltimore, Maryland, USA.

Contador, J.F.L., Schnabel, S., Gutiérrez, A.G., FernUndez, M.P., 2009. Mapping sensitivity to land degradation in Extremadura, SW Spain. Land Degrad. Dev., 20, 129-144.

DRC staff (1984). Internal report for the scientific program on soil studies at El-Bahariya Depression. South Valley, Egypt.

Elnaggar, A.A., 2014. Spatial Assessment of Groundwater Quality for Irrigation in Bahariya Oasis, Egypt. Journal of Soil Science, ISSN: 2231-6833 \& E-ISSN: 2231-6841, 3 (1), 034-039.

Elnaggar, A.A., 2017. Development of Land Capability and Suitability Maps for Bahariya Oasis, Egypt. Egypt. J. Soil Sci., 57 (4), 489-503 .

El-Shazly M.M., El-Ramly I.M., Guindy Kh.A., Abdel Aati A., 1991. Bahariya formation water quality characteristics and its relationship to the hydrothermal activity in Bahariya Oasis, Western Desert, Egypt. Geol. Soc. Egypt. $29^{\text {th }}$ Annual Meeting, Cairo, Egypt.

El-Sisi, Z., Hassaouba, M., Odani, M.J., Dolson, J.C., 2002. The Geology of Bahariya Oasis in the Western Desert of Egypt and its Archeological Heritage, Field Trip No. 8, Cairo International Conference and Exhibition, October 27-30.

EMA, 2012. Egyptian Meteorological Authority (EMA), Annual reports, Cairo, Egypt.

Fantechi, R., Peter, D., Balabanis, P., Rubio, J. L., 1995. In R. Fantechi, D. Peter, P. Balabanis, \& J. L. Rubio (Eds.), Desertification in a European context: Physical and socio-economic aspects. Brussels, Belgium: Office for Official Publications of the European Communities, EUR 15415. 
FAO, 2006. Guidelines for Soil Description, $4^{\text {th }}$ Edition. Food and Agriculture Organization of the United Nations, Rome, Italy.

Gao, J., Liu, Y., 2008. Mapping of land degradation from space: a comparative study of Landsat ETM+ and ASTER data. Int. J. Remote Sens., 29, 4029-4043.

Hamdan, M., 2012. Multivariate statistical analysis of geochemical data of groundwater in El-Bahariya Oasis,Western Desert, Egypt; Res. J. Environ. Earth Sci. 4(6): 665-667.

Hamdan, M., Sawires, F., 2013. Hydrogeological studies on the Nubian sandstone aquifer in El-Bahariya Oasis,Western Desert, Egypt; Arab. J. Geosci. 6(5): 1333-1347, doi: 10.1007/s12517-011-0439-8.

Khalifa, M. E.A., 2016. Monitoring of Environmental Sensitivity to Desertification in Some Areas at West of Delta, Egypt. Zagazig J. Agric. Res., 43 (3): 901-921.

Khalifa, M.A., Catuneanu, O.,Wanas, H.A.,2006. Sequence stratigraphy of the lower Cenomanian bahariya formation, Baha-riya Oasis, Western Desert, Egypt, Geology Dept., Fac. of Sci., Menoufia Univ., Shebin El-Kom, Egypt, 190(1-4), 121-137.

Korany E.A., 1984. On the demonstration of the hydrogeological control by local geologic structures, Bahariya Oases, Egypt. E.G.S. Proc. of $3^{\text {rd }}$ Annual Meeting, 341-335.

Kosmas, C., Ferrara, A., Briasouli, H., Imeson, A., 1999. Methodology for mapping environmentally sensitive areas (ESAs) to desertification. In: Kosmas, C., Kirkby, M., Geeson, N. (Eds.), The Medalus Project: Mediterranean Desertification and Land Use. Manual on Key Indicators of Desertification and Mapping Environmentally Sensitive Areas to Desertification. European Union 18882.

Kosmas, C., Tsara, M., Moustakas, N., Karavitis, C., 2003. Identification of indicators for desertification. Ann. Arid Zones, 42: 393-416.

Masoud, M.H., El-Osta, M.M., 2016. Evaluation of groundwater vulnerability in El-Bahariya Oasis, Western Desert, Egypt, using modelling and GIS techniques: A case study. J. Earth Syst. Sci., 125 (6),1139-1155. DOI 10.1007/s12040-016-0725-7.

Montanarella, L., 2007. Trends in land degradation in Europe. In M. V. Sivakumar, \& N. N'diangui (Eds.), Climate and land degradation. Berlin: Springer. 
Peterson, F.F. 1981. Landforms of the Basin and Range Province: Defi ned for soil survey. Tech. Bull. 28. Nevada Agric. Exp. Stn., Univ. of Nevada, Reno.

Rasheed, M.A., Gad, A., Zoelitz-Moeller, R., Darwish, Kh.M., 2008. Geographic Mapping and Analysis Using GIS of Study Areas in Bahariya Oasis, Egypt. American-Eurasian J. Agric. \& Environ. Sci., 4 (1): 125-130.

Rasheed, M.A., Wahab, M.A., Youssef, R.A., 2010. Digital Geopedological Mapping of Some Study Areas in Western Desert, Egypt. Journal of American Science, 6(9), 23-29.

Salvati, L., Bajocco, S., 2011. Land sensitivity to desertification across Italy: Past, present, and future. Applied Geography, 31, 223-231.

Salvati, L., Zitti, M., 2008. Regional convergence of environmental variables: empirical evidences from land degradation. Ecological Economics, 68: 162-168.

Santini, M., Caccamo, G., Laurenti, A., Noce, S., Valentini, R., 2010. A multi-component GIS framework for desertification risk assessment by an integrated index. Appl. Geogr., 30: 394-415.

Sepehr, A., Hassanli, A.M., Ekhtesasi, M.R., Jamali, J.B., 2007. Quantitative assessment of desertification in south of Iran using MEDALUS method. Environ. Monit. Assess., 134, 243-254.

Shehata M.A., 1992. Geohydrological studies on the Central part of Western Desert, Egypt, Ph.D. Thesis, Faculty of Sci-ence, Zagazig University, Egypt, 268.

Soil Science Division Staff, 2017. Soil survey manual. United States Department of Agriculture (USDA), Agriculture Handbook No. 18. Natural Resources Conservation Service, Washington, D.C.

Soil Survey Staff, 2014. Kellogg Soil Survey Laboratory Methods Manual. Soil Survey Investigations Report, No. 42, version 5.0. R. Burt and Soil Survey Staff (eds.). Washington DC: U.S. Department of Agriculture-Natural Resources Conservation Service.

UNEP (2003). United Nations Environment Programme. Egypt Human Development Report. Cairo, Egypt, 166 pp.

Zahran M.A., Willis A.J., 2009. The Vegetation of Egypt, $2^{\text {nd }}$ ed., Springer Science Business Media B.V., 44. 


\section{التقييم الكمى للتصحر فى بيئة واحة البحرية ، الصحراء الغربية ، مصر}

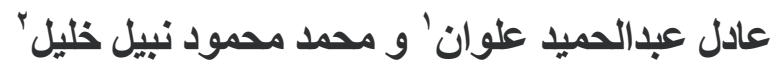

$$
\begin{aligned}
& \text { ' قسم البيدولوجى، شعبة مصادر المياه و الأراضى الصحر اوية، مركز بحلئ بحوث }
\end{aligned}
$$

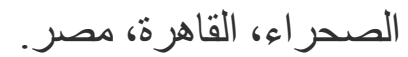

$$
\begin{aligned}
& \text { × قسم علوم الار اضى و المياه ، كلية الزراعة ، جامعة الزقازيق، مصر }
\end{aligned}
$$

التصحر هو أحد أهم الششاكل التي تواجه الهناطق الجافة وشبه الجافة حول

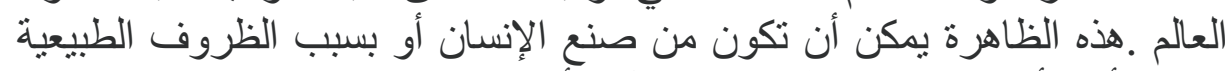

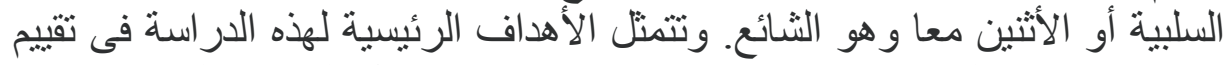

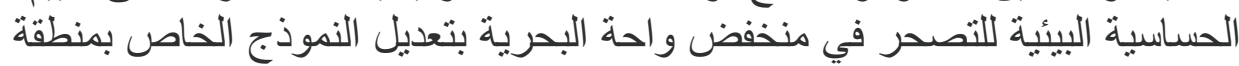

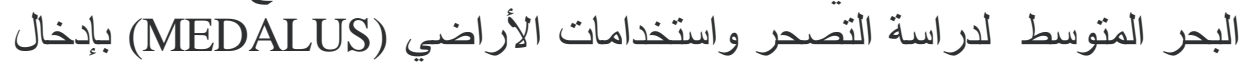

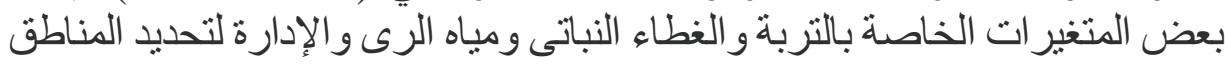

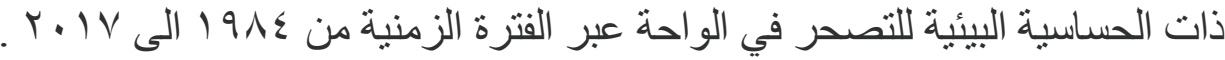

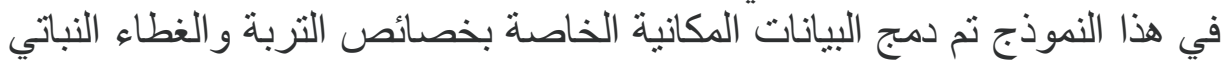

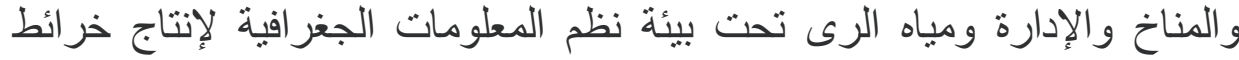

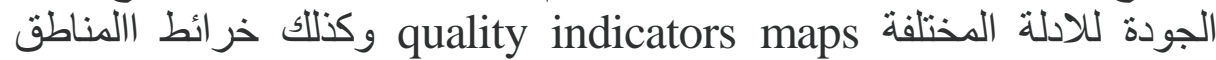

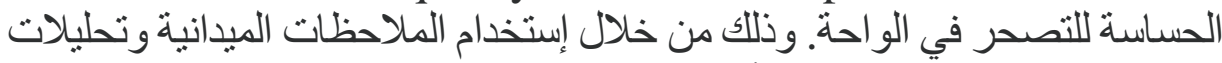

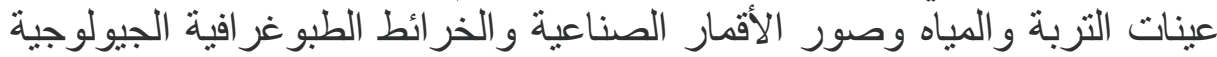

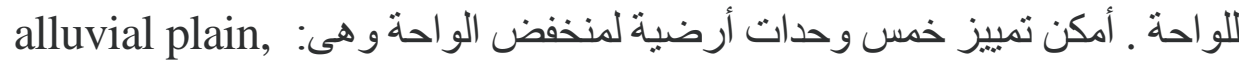
. lowland, peneplain, plateau and hills, playa

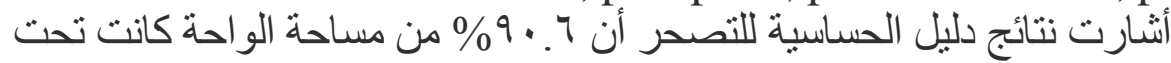

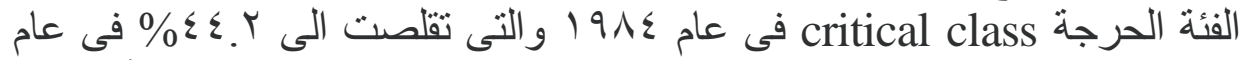

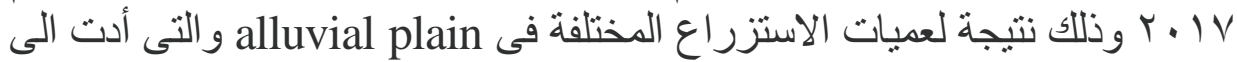

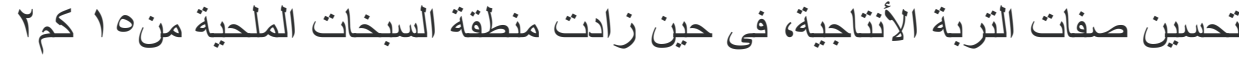

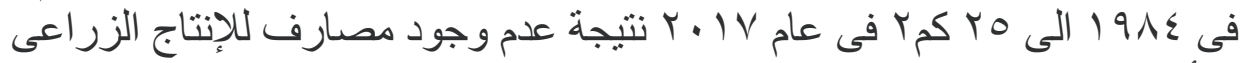
بالأر اضى المنخفضة بالو احة و التى تتعلق بالإدارة السيئة بتلك بلك المنطقة.

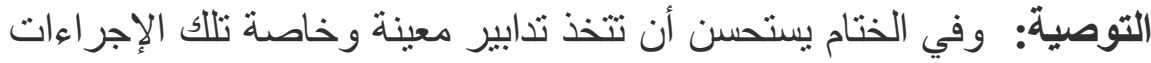

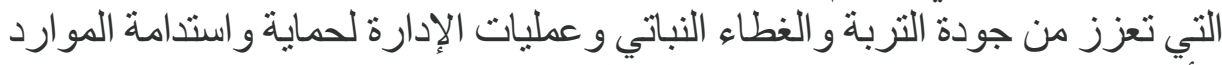
الأرضية في منخفض جردة واحة البحرية ضد النصاء التصحر. 